\title{
Organización del conocimiento en la red digital
}

\author{
Mary Eugenia Luna González *
}

Artículo recibido:

5 de diciembre de 2013.

Artículo aceptado:

9 de octubre de 2014.

\section{Resumen}

Se realiza un análisis documental que permite conocer el estado de la organización del conocimiento en la documentación encontrada en la red digital, partiendo del análisis del conocimiento dentro de dichos sistemas informativos. Intervienen en ello el contexto sociocultural en el que se desenvuelve el creador del conocimiento y el conjunto de valores y creencias que comparte el personal que lo representa, así como las necesidades informativas de los usuarios finales de la información que es resultado de la organización del conocimiento. De igual manera se aborda la relación existente entre el usuario y los sistemas de recuperación del conocimiento en la red digital.

* Ministerio de Petróleo y Minería, Venezuela. malunag2000@yahoo.com

INVESTIGACIÓN BIBLIOTECOLÓGICA, Vol. 29, Núm.67, septiembre/diciembre, 2015, México, ISSN: 0187-358X. pp. 77-89 
Palabras clave: Organización del Conocimiento; Contexto; Red Digital; Recuperación del Conocimiento; Representación.

\section{Abstract \\ Organization knowledge on the digital network Mary Eugenia Luna-González}

This paper presents a documental analysis for the purpose of understanding how knowledge in digital document repertories is organized, specifically those hosted on the digital network. The study is carried out on the basis of an analysis of the knowledge within these information systems, in which the sociocultural context of the creator and the subset of values and beliefs shared by the personnel representing this creator necessarily intervene in conjunction with the informational needs of the end user of the information product offered by the knowledge organization. The paper also discusses the relationship existing between the user and the digital network knowledge retrieval system.

Keywords: Organization of the Knowledge; Context; Digital Network; Recovery of the knowledge; Representation.

\section{INTRODUCCIÓN}

L as transformaciones y el avance de las tecnologías de la información han determinado la manera en que, en el transcurso de los años, ha evolucionado la forma en que se crea, registra y se accede al conocimiento. La organización del conocimiento se encarga de representarlo utilizando lenguajes documentales por medio de conceptos y terminologías, controladas o no; dichos lenguajes dependen de la realidad de los profesionales encargados de representar dicho conocimiento, y del contexto sociocultural en el que se desenvuelven. Los diferentes contextos están determinados por las condiciones económicas, políticas y socioculturales del entorno, lo cual hace que existan diferentes formas de analizar, interpretar y representar el conocimiento. El trabajo de representación del conocimiento es el proceso que realiza el do- 
cumentalista al mediar entre la información producto del conocimiento y el usuario final.

Con base en lo anterior, el objetivo del presente trabajo es conocer cómo se lleva a cabo la organización del conocimiento en los repertorios documentales, específicamente los encontrados en la red digital. La metodología empleada consiste en el análisis documental, examinando la literatura especializada en el área y que incluye fuentes documentales tanto impresas como digitales, para elaborar un marco conceptual relacionado con el tema de estudio; con ello se obtiene un dominio terminológico que permitirá emitir conclusiones sobre el tema de organización del conocimiento en la red digital.

\section{OrganizaCión DEL CONOCIMIENTO}

La organización del conocimiento como disciplina dentro de la ciencia de la información se encarga de estudiar el tratamiento y la recuperación del conocimiento, así como la construcción y control de los lenguajes e instrumentos utilizados en los procesos de representación de los documentos que son producto del conocimiento humano, permitiendo así su posterior recuperación por parte de los usuarios. Dicha organización se concibe como un proceso en el que el documentalista o el profesional en el área de información intermedia entre el productor documental y el usuario final del conocimiento por medio de la representación del mismo.

En el sentido más específico, los bibliotecólogos, archivólogos y especialistas que organizan el conocimiento realizan actividades como la descripción de documentos, indizaciones, bases de datos bibliográficas, archivos y otros tipos de "Memorias de Instituciones" (Hjørland, 2008: 86). Respecto de tales actividades en relación con los documentos, la información y el conocimiento, Vizcalla Alonso establece que

[La] organización de la información, se ha relacionado directamente con la conocida etapa del llamado procesamiento de la información y se ha enmarcado dentro de los aspectos tradicionales del mismo que, de forma genérica, se pueden resumir como todo lo relacionado con la descripción de la forma y el contenido de los documentos que entran a formar parte del sistema. (1997: 166)

La organización del conocimiento establece los sistemas de representación de la realidad del autor de dicho conocimiento, con la finalidad de que el usuario final pueda acceder y comprender el contexto en el que fue creado un conocimiento específico. Estos sistemas contemplan los lenguajes docu- 
mentales, tal como menciona Civallero (2005: 1): "[los lenguajes documentales] se transforman, así, en un intermediario entre la información y el usuario, codificando tanto el contenido de un texto como la búsqueda del lector". Por su parte, Gil Urdiciain (1996: 1) establece como lenguaje documental "todo sistema artificial de signos normalizados, que facilitan la representación formalizada del contenido de los documentos para permitir la recuperación, manual o automática, de información solicitada por los usuarios".

$\mathrm{Al}$ ser el lenguaje documental un intermediario entre la información y el usuario que permite la recuperación de información, se encuentra cargado de la subjetividad inherente al ser humano ya que las representaciones realizadas mediante el lenguaje documental se encuentran inmersas en un contexto y responden a las ideologías de los organizadores del conocimiento.

\section{EL CONTEXTO Y LA IDEOLOGÍA EN LA ORGANIZACIÓN DEL CONOCIMIENTO}

Los documentalistas, o el personal encargado de representar el conocimiento, realizan una tarea de mediación entre la documentación y el usuario final, utilizando para ello lenguajes controlados que pretenden garantizar neutralidad pero que en realidad ofrecen registros y resúmenes informativos cargados de la subjetividad inherente al ser humano, proveniente de las experiencias previas de dichos profesionales y del entorno en el que se desenvuelven.

El contexto juega un rol fundamental en la descripción y representación de la información escrita pues involucra aspectos sociales y culturales. El lenguaje empleado en el discurso de los autores del conocimiento y de sus usuarios finales varía de acuerdo con las ideologías y la relación con el entorno. Por consiguiente, como indica Moreiro (citado en Bufrem, Silva y Breda, 2005: 124):

Las tareas de selección y atribución de términos por los profesionales de la información, más allá de estar inspiradas en las necesidades de los usuarios, se basan principalmente en el contexto de las culturas a las cuales pertenecen así como en la experiencia de los interlocutores. Dichas tareas se llevan a cabo dentro de un universo de posibilidades para la representación de los conceptos seleccionados, que abarcan desde vocabularios controlados hasta lenguajes libres, pasando por las posibles circunstancias que llevan a la decisión de incluir un término como representante del contenido original.

$\mathrm{Al}$ respecto, Van Dijk (citado en Silva, 1997) indica que en todos los niveles del discurso se encuentran las huellas contextuales, en las que las características sociales de los participantes, tales como género, clase, etnicidad, 
edad, origen, posición u otras formas que indiquen la pertenencia a un grupo, juegan un rol fundamental o vital en la representación. De esta forma, el paradigma físico exige al profesional mediador que el tratamiento a la información y el lenguaje de representación sean de carácter científico y controlado, aunque el lenguaje y estructuración de la fuente primaria no lo sea. No obstante, aunque así lo decidan, por la propia naturaleza humana dichos profesionales no pueden limitarse a realizar simples transcripciones neutrales y objetivas, imprimiendo así a las representaciones características de sus realidades particulares. Tal actividad contradice en la práctica a la premisa que indica que el conocimiento y la información deben organizarse en función del usuario que la recupera para su uso posterior, decidiendo qué es relevante y qué no lo es.

De igual manera, la situación en donde se confiere neutralidad a la representación del conocimiento por medio de la utilización de lenguajes controlados parte de los lineamientos rígidos del paradigma físico, en el cual "la crisis paradigmática se evidencia (para ilustrar con un ejemplo) en el desencuentro tajante entre investigadores y prácticos que consideran que el trabajo de nuestros profesionales es neutral e inocuo" (García Gutiérrez, 2001: 3 ).

Tomando en cuenta además que la ideología determina el lenguaje utilizado por el autor y el mediador, se puede decir que está entendida como un conjunto de valores y creencias que comparten los integrantes de una determinada sociedad y que es asimilada por los miembros de una manera inconsciente, convirtiéndose en la concepción de realidad de los integrantes de un grupo y a su vez modelando una identidad y conformando una personalidad (Civallero, 2005).

Dicho esquema ideológico estructura la opinión de las personas sobre una amplia gama de temas específicos, configurando también la manera en que actúan; esto puede resultar problemático cuando se genera una actitud negativa hacia un sector en particular. Al presentarse las connotaciones negativas de las ideas, pueden llevar a dichos grupos a actuar de manera perjudicial y discriminativa hacia los grupos a los que les otorgan tales connotaciones.

Como indica Civallero (2005: 3), en diversas herramientas de representación con lenguaje controlado se evidencia que tales connotaciones negativas se consideran objetivas:

En una herramienta de trabajo documental usada a nivel internacional, se han incluido descriptores controlados por profesionales, representativos, unívocos y pertinentes, que clasifican a pueblos y razas enteras como "coloniales" o como "primitivos" (en oposición a pueblos "desarrollados" o "altamente desarrollados”, términos también incluidos). Esta clasificación implica establecer etiquetas y diferencias mentales ( $\mathrm{y}$ físicas) que profundizan distancias entre seres humanos. 
Se perpetúan, de esta manera, políticas de odio, marginación, dominio, desprecio y violencia, que han marcado tristemente (y aún marcan) las páginas de la historia humana. Pero quizás lo más preocupante sea el hecho de que estas etiquetas, estos descriptores, han sido aceptadas como "neutrales" por la sociedad que generó este lenguaje documental. Es más, estos términos son considerados necesarios, pertinentes, importantes y objetivos para la clasificación y la comprensión de ciertos grupos humanos.

Así, en vista de que los profesionales que representan la información no pueden limitarse a realizar simples transcripciones neutrales y objetivas en tanto que las características de sus realidades particulares se ven reflejadas en sus representaciones, se contradice la premisa que indica que el conocimiento y la información deben organizarse en función del usuario, quien al recuperar la información generalmente accede a sistemas digitales, disponibles en la red, en donde el que representa la información es el que decide qué es relevante o no.

\section{LA ORGANIZACIÓN DEL CONOCIMIENTO EN LA RED DIGITAL}

Con la aparición y evolución de las tecnologías de la información y comunicación, las formas de representar y organizar el conocimiento y los procesos de registro y de acceso al mismo han sufrido transformaciones.

Una de esas tecnologías es la red digital, invención mecanicista creada con la finalidad de controlar el crecimiento acelerado del conocimiento; es entendida como un sistema de información abierto en el que el ingreso y actualización del conocimiento en los sistemas de representación permite la interacción y publicación documental de diversos individuos, tanto especialistas como aficionados a temas específicos, lo que trae como consecuencia una saturación informativa y sobrecarga del sistema digital.

En tal sentido, la organización del conocimiento en las redes electrónicas ofrece el acceso en una sola plataforma de manera directa. Puesto que el conocimiento circulante en los sistemas informativos está conformado por mediaciones y codificaciones de la producción real, ésta se descontextualiza, debiendo el usuario aprender a seleccionar y refinar los términos de búsqueda hasta encontrar la información relevante que realmente necesita.

Es por ello que el trabajo de selección llevado a cabo por los documentalistas tiene como finalidad el análisis documental para representar y difundir, por medio de soportes materiales y tecnológicos, la información que sirve de fuente a los usuarios. Chacón Gutiérrez (1995) indica que dicho proceso es abstracto (utiliza una serie de códigos en el sistema de almacena- 
miento, búsqueda y posterior recuperación) y anónimo (el analista y el usuario final no se conocen entre sí y desconocen sus necesidades mutuas). Así, el usuario delega su búsqueda en otro individuo del cual ignora el modo y las razones que lo motivan a actuar de determinada manera.

El procedimiento abstracto de la organización del conocimiento en la Web, en donde se asignan códigos o metadatos al documento para hacerlo más explícito y fácil de representar, indizar y posteriormente buscar, carece de procedimientos normalizados en la asignación de tales caracteres, lo que puede resultar, como productos, representaciones no tan completas. Codina indica al respecto que "[...] las personas nos equivocamos, y los autores de las páginas web se equivocan: se olvidan de poner metadatos, los ponen mal, lo hacen en unas páginas sí y en otras no, se equivocan en la ortografía [...]" (2003: 151).

En síntesis, la indización de la producción intelectual y del conocimiento en dichos servidores es realizada por un gran número de expertos encargados de seleccionar, simplificar y representar la documentación producto del trabajo intelectual que los especialistas y autores publican sobre un tema específico, lo que trae como consecuencia que dicha información sea distorsionada o manipulada por parte del personal que realiza la mediación, cambiando así los conceptos originales al interactuar en un contexto diferente al de los productores del conocimiento y afectando el proceso de búsqueda del usuario al momento en que se arrojan los resultados.

\section{LA ORGANIZACIÓN DEL CONOCIMIENTO EN LOS SISTEMAS DE RECUPERACIÓN DE INFORMACIÓN DIGITAL}

Los resultados que arrojan los buscadores de Internet son producto de la coincidencia de elementos textuales, mas no de contenido ni de las relaciones de dichos términos, careciendo entonces de un mecanismo de recuperación lógica por estar desprovisto del sentido semántico. Esto lleva a que las búsquedas informativas de los usuarios ocasionen que el sistema enlace términos que sobresaturan y obstruyen la recuperación informativa, haciendo que los resultados no tengan relación informativa con las temáticas buscadas y mezclando lo fundamental con lo irrelevante, además de recuperar información inapropiada o carente de sentido para el usuario que pretende cubrir una necesidad. En consecuencia, el usuario abandona la búsqueda al considerar a tales sistemas electrónicos carentes de respaldo científico e incapaces de cubrir demandas informativas en una inversión óptima de tiempo y esfuerzo. 
Las indizaciones elaboradas por el personal documentalista que labora en las compañías de recuperación de información electrónica a través de Internet no hacen distinción entre los usuarios a los que prestan sus servicios por no conocerlos potencialmente; por su parte, el usuario consigue información que no ha sido discriminada según sus necesidades al tener libre acceso al contenido de dichas bases de datos. Entonces, en palabras de García Gutiérrez (2001: 6), "no innovan en las lógicas de organización del conocimiento, mantienen conservadoramente las estructuras clásicas, y es el propio soporte quien decide los cambios".

Con respecto a los lenguajes documentales utilizados para indizar la documentación en los sitios de búsquedas electrónicas, pueden ser manipulados por algunas de las siguientes razones (Chacón Gutiérrez, 1995: s. pág.):

1. El analista, al igual que el creador del lenguaje documental, se encuentra inmerso en una sociedad que impone modelos ideológicos y culturales.

2. En las operaciones intelectuales la subjetividad del mediador documental influye en la selección de la temática de los documentos y en la elección de palabras claves o descriptores utilizados en la representación.

3. El lenguaje documental es codificado, y aunque el usuario y el documentalista intermediario deben compartir los códigos documentales, lo cierto es que dichos códigos están condicionados por el personal que realiza la mediación.

4. La indización, como proceso en donde se traduce del lenguaje natural al documental, puede sufrir de manipulación al no reflejar las connotaciones implícitas en el lenguaje original ni el contexto sociocultural en el que se produjo. De igual manera, la falta de información del que indiza puede influir negativamente en la elección de los conceptos y palabras claves presentes en la documentación.

Como menciona García Gutiérrez (2001: 5-6): "por el momento, el hipertexto no va más allá de los viejos índices sistemáticos o permutados, no es más que un índice transversal y caótico con efectos especiales".

La interacción usuario-sistema en las búsquedas informativas es más productiva cuando el usuario es capaz de plantear sus necesidades informativas, coincidiendo éstas con el lenguaje de representación utilizado en la indización, aunque es necesario indicar que si el usuario realiza sus indagaciones utilizando los términos del vocabulario controlado del sistema se ve limitado a su vez por las características del mismo, ya que en los sistemas electrónicos de recuperación del conocimiento en la red se utilizan diversos procedimientos para llevar a cabo las búsquedas. Uno de ello es la recuperación por medio de la búsqueda lógica, en donde se utilizan operadores booleanos, relacionando y combinando los términos de una manera lógica. Se utilizan generalmente las relaciones AND, OR y NOT. 
La relación AND (conjunción o intersección lógica) es utilizada para relacionar dos o más términos que se requiere que aparezcan a la vez en los documentos. Con la relación OR (suma lógica) se buscan documentos que contengan uno de los términos o todos ellos. La relación NOT (negación lógica) excluye los documentos en los que aparezca un término determinado (Lancaster y Pinto, 2001).

De igual manera, dentro de los sistemas de recuperación de información se realiza la búsqueda utilizando el texto libre, con palabras y frases en lenguaje natural, lo cual arroja la coincidencia de términos en el título del documento o en cualquier parte de éste. En tal procedimiento la estrategia para lograr la búsqueda está conformada por las frases que el usuario considera pertinentes para recuperar el conocimiento de acuerdo a su necesidad.

La ventaja que brinda la libre utilización del lenguaje natural en las búsquedas es poder realizarlas de una manera más específica que utilizando lenguajes controlados, pero a su vez genera resultados con frases del usuario que coinciden con el lenguaje documental de los profesionales, quienes realizan representaciones subjetivas que interfieren con la neutralidad en la organización del conocimiento.

\section{Neutralidad en la ORganización del CONOCIMIENTO EN LA RED DIGITAL}

Existe una relación entre el contexto sociocultural y la expresión del conocimiento representada en el discurso documental, tal relación es construida por los participantes de dicho discurso, teniendo cada persona una interpretación particular del contexto en el que se encuentra inmerso.

El personal que procesa los contenidos documentales en la Web no realiza distinciones entre los usuarios a los que presta sus servicios; como el usuario tiene libre acceso al contenido de dichas bases de datos consigue información que no ha sido discriminada según sus necesidades. Lo anterior obedece a que los profesionales de la información no pueden realizar descripciones ni transcripciones neutrales y objetivas, por lo que imprimen en sus representaciones la carga subjetiva inherente a la realidad y al contexto en el que se desenvuelven.

En tal sentido, Chacón Gutiérrez (1995: s. pág.) menciona respecto a la descripción e indización:

Al ser una operación intelectual la subjetividad del analista opera de forma decisiva en la selección de los temas que contiene un documento y en la elección de descripto- 
res o de palabras-clave que los representan. El documentalista actúa como lector-destinatario del documento primario, interpreta el documento que recibe y transmite su contenido (emisor-intermediario) traducido a un lenguaje codificado (co-autor).

Es por ello que el profesional de la documentación, al realizar los análisis documentales e indizaciones en la Web, simplifica y representa el trabajo de los autores sobre un tema en específico, poniéndolo al servicio y disposición de los usuarios de la Web. En consecuencia, al procesar la información puede suceder que sea distorsionada o manipulada al cambiar términos y conceptos para adecuarlos a su realidad y a su contexto. Como indica Chacón Gutiérrez (1995: s. pág.) con respecto a la manipulación en la indización, que corresponde a una traducción de lenguaje natural al documental:

El documento secundario, traducido del primario, es un intermediario entre el usuario y el original, si éste ha sido manipulado en la indización el usuario será manipulado. La manipulación puede proceder de los problemas no resueltos que atañen a toda traducción: falta de fidelidad al original; no encontrar equivalencias entre la lengua natural y el lenguaje documental; no reflejar las connotaciones implícitas en el original, ni el contexto sociocultural en el que se escribió; que la subjetividad, o la falta de formación del indizador, influyan negativamente en la elección de los conceptos presentes en el documento o de las palabras clave o descriptores que los representan.

El documentalista, influenciado por sus cogniciones sociales, lee el documento primario asumiendo normas, valores, objetivos e intereses socialmente compartidos; a partir de ello efectúa el proceso documental y construye el documento secundario, convirtiéndose de esa manera en el coautor de la información final que se pondrá a disposición del usuario. "Los datos ofrecidos por un Centro de Documentación no suelen ser verificados por los usuarios, el usuario se somete así a una doble manipulación, la del documento secundario y la del documento primario en que tiene su origen" (Chacón Gutiérrez, 1995: s. pág.). De la misma manera señalan Bufrem, Silva y Breda (2005: 123):

[...] cuando el mediador o el lector interlocutor interpreta el texto para representarlo debe concebirlo como un texto socialmente dirigido y situado en un momento preciso y debe aceptar que la estructura de la enunciación está determinada por la situación social más inmediata y el medio social más amplio.

En cuanto a las búsquedas, en el entorno digital se encuentra documentación que para poder ser recuperada por el usuario ha sido representada en el buscador, utilizando para ello palabras que han sido elegidas de un grupo 
disponible pertenecientes a un conjunto social. Por lo tanto, los procesos de selección de conceptos por parte de los documentalistas o profesionales de la información dentro del sitio web de búsquedas se encuentran basados en el contexto sociocultural y en las experiencias de tales especialistas más que en las necesidades informativas de los usuarios. Tal como indica Moreiro (citado en Bufrem, Silva y Breda, 2005), tales tareas son llevadas a cabo dentro de un universo de posibilidades para la representación de los conceptos seleccionados, alcanzando desde lenguajes controlados hasta libres, y atravesando por circunstancias que determinan si se incluye un término u otro para representar el conocimiento (Moreiro, citado en Bufrem, Silva y Breda, 2005).

Finalmente, cuando el usuario realiza una búsqueda de información, la coincidencia de conceptos (mas no de contenidos) en los sistemas de recuperación electrónica arroja como resultado información irrelevante, obviando contenidos pertinentes en la investigación o en la búsqueda simple e interfiriendo en la neutralidad de la información representada.

\section{Conclusiones}

1. La representación del conocimiento es la manifestación del pensamiento que se expresa a través del lenguaje, el cual permite descifrar cómo los individuos reproducen el pensamiento en su pertenencia a un grupo social o cultural específico.

2. La organización del conocimiento es un campo que desempeña un papel fundamental en el ámbito del intercambio y acceso informativo al conocimiento en general. Se apoya en las bondades ofrecidas por las redes tecnológicas al momento de recuperar la información representada en dichos medios, pero es necesario destacar que el papel de selección terminológica y de contenido dentro de estos sitios debe ser llevado a cabo por profesionales en el área de las ciencias de la información.

3. Los participantes del discurso documental construyen la relación existente entre el contexto sociocultural y la expresión del mismo, interpretando cada individuo de manera particular el contexto en donde se desenvuelve e imprimiendo en sus representaciones características de sus realidades particulares; en consecuencia, tal representación del conocimiento no es del todo neutral.

4. Es necesaria la intervención de instancias que controlen las herramientas de representación documental en los casos de connotaciones conceptuales negativas en la representación del conocimiento que 
pretendan pasar como formas neutras, naturales y correctas de indizar la información, pues contienen juicios valorativos discriminatorios hacia grupos socioculturales específicos.

5. Las actividades tradicionales de origen humano están siendo realizadas cada vez más por medios computarizados; los profesionales en el área de información se concentran en aplicar las tecnologías de la información y comunicación y dejan en segundo lugar el trabajo de interpretación y análisis del significado y contenido del conocimiento.

6. Con la llegada de Internet se intensificaron las relaciones entre el sujeto productor del conocimiento y el usuario final del mismo por medio de las exploraciones realizadas en los buscadores digitales. En las búsquedas se utilizan lenguajes tanto normalizados como libres por parte del responsable que indiza, quien se encuentra inmerso en su contexto personal, lo cual incide en que los resultados arrojados sean coincidencia de palabras mas no de contenido según las necesidades del usuario.

7. El empleo de operadores booleanos en las búsquedas de conocimiento en los sistemas digitales de recuperación de información, a pesar de ofrecer ventajas en cuanto a la combinación terminológica y la inclusión o exclusión de palabras según sea indicado por cada operador, presenta una situación desfavorable cuando no todas las personas que recurren a dicho servicio se encuentran familiarizadas con su correcta combinación, lo cual hace que los sistemas arrojen información errónea o innecesaria.

8. En los sistemas digitales de información interactivos con el usuario se manifiestan problemas cuando éste, por desconocimiento, no representa adecuadamente su necesidad informativa mediante los operadores booleanos, condenando la búsqueda al fracaso u obteniendo información irrelevante de acuerdo al tema indagado.

9. Las búsquedas libres pueden ser más específicas que las realizadas mediante lenguajes controlados, pero a su vez generan dificultades cuando inciden en la neutralidad al momento de la recuperación mostrando información irrelevante y obviando contenidos pertinentes en la investigación.

10. La organización del conocimiento en la Web se encuentra amenazada cuando el personal del área de información encargado de indizar el conocimiento desconoce las necesidades de los usuarios de la red, y por ende los análisis se ven cargados de elementos subjetivos provenientes del contexto y de las diferentes realidades. 


\section{REFERENCIAS BIBLIOGRÁFICAS}

Bufrem, L. S.; Silva H. F.; Breda S. M. (2005), "Reformulación de los elementos teóricos de la organización del conocimiento: bases lingüísticas y culturales y estructuras de representación”, en ISKO, capítulo español. Congreso $7^{\circ}$, Barcelona, pp. 121-127.

Chacón Gutiérrez, I. (1995), "La mediación documental”, en Cuadernos de Documentación Multimedia, vol. 4. Disponible en: http://www. ucm.es/info/multidoc/multidoc/revista/cuadern4/chacon.html [Fecha de consulta: 10 de enero de 2011].

Civallero, E. (2005), "Espinas ocultas: lenguajes documentales, ideologías negativas y revisiones”, en Fuentes del Congreso. Boletín de la Biblioteca y Archivo Histórico del H. Congreso Nacional de Bolivia, vol. 4, núm. 20. Disponible en: http://issuu.com/bib.csinfo/ docs/civallero__2005 [Fecha de consulta: 10 de enero de 2011].

Codina, L. (2003), "La web semántica: una visión crítica", en El Profesional de la Información, vol. 12, núm. 2, marzo-abril, pp. 149-152.

García Gutiérrez, A. (2001), "Redes digitales y exomemoria”, conferencia presentada en I Congreso Ibérico de Comunicación. La Sociedad de la Comunicación en el siglo xxi, Universidad de Málaga, mayo de 2001, Málaga.

Gil Urdiciain, B. (1996), Manual de lenguajes documentales, Madrid: Noesis.

Hjørland, B. (2008), "What is Knowledge Organization (KO)?", en Knowledge Organization, vol. 35, núm. 2-3, pp. 86-101.

Lancaster, F. (1995), El control del vocabulario en la recuperación de información, Valencia: Universidad de Valencia.

Lancaster, F.; Pinto, M. (2001), Procesamiento de la información científica, Madrid: Arcolibros.

Silva, O. (1997), "El estudio del discurso en el camino de Teun Van Dijk", en Revista Frontera, Universidad de la Frontera, Temuco, vol. 16, pp. 97-106.

Sojo, V. (2008), Normas de la American Psychological Association (APA) para las citas y referencias bibliográficas, recopilación y traducción, Caracas: Escuela de Psicología, UCV.

Van Dijk, T. (2001), "Algunos principios de una teoría del contexto", en Revista Latinoamericana de Estudios del Discurso, vol. 1, núm. 1, pp. 69-81.

Vizcalla Alonso, D. (1997), Información: procesamiento de contenido, Argentina: Parhadigma Ediciones. 